\title{
Cirugía bariátrica secundaria en el Hospital Universitario San Ignacio
}

\author{
Secondary bariatric surgery at San Ignacio University Hospital \\ Rami Mikler ${ }^{1} \mathbb{D}$, Fabio Eduardo Pinzón C. ${ }^{1} \mathbb{D}$, Ricardo Metke ${ }^{2}$ \\ 1 Médico, especialista en Cirugía general y Cirugía bariátrica, Clínica de obesidad, Departamento de Cirugía, Hospital Universitario \\ San Ignacio, Pontificia Universidad Javeriana, Bogotá, D.C., Colombia. \\ 2 Médico, residente de Cirugía general, Pontificia Universidad Javeriana, Hospital Universitario San Ignacio, Bogotá, D.C., Colombia.
}

\section{Resumen}

Introducción. La Organización Mundial de la Salud define la obesidad como un incremento en el porcentaje de grasa corporal, generalmente acompañado de aumento en el peso, cuya cantidad y distribución condicionan la salud del individuo. Se caracteriza por ser una enfermedad crónica y de muy difícil manejo. La cirugía bariátrica es un procedimiento cada vez más frecuente, que ha demostrado ser la mejor opción terapéutica para el manejo de la obesidad moderada y severa, enfermedad que ha ido ganado terreno en el mundo. Según la Encuesta Nacional de la Situación Nutricional en Colombia 2015, la incidencia de sobrepeso fue del 37,8 \% y de obesidad del 18,7 \%. Con el incremento de la cirugía bariátrica primaria, también se ha incrementado la frecuencia de la cirugía bariátrica de revisión, conversión y reversión, siendo la de conversión la más frecuente.

Métodos. Estudio observacional retrospectivo en el que se analizaron los pacientes llevados a cirugía bariátrica de conversión en el Hospital Universitario San Ignacio entre 2014 y 2019.

Resultados. En nuestra institución la cirugía de conversión corresponde al 8,8 \%, y coincide con la estadística mundial para este procedimiento. La causa más frecuente, a diferencia de la literatura, es el reflujo gastroesofágico, seguido de la reganancia de peso.

Discusión. Los resultados obtenidos en el Hospital Universitario San Ignacio se asemejan a las cifras mundiales de procedimientos de conversión. Se obtiene la resolución de los síntomas de reflujo gastroesofágico y el manejo adecuado de la reganancia de peso en un alto porcentaje de pacientes, con muy pocas complicaciones.

Palabras clave: obesidad; cirugía bariátrica; secundaria; conversión; reflujo gastroesofágico; pérdida de peso.

Fecha de recibido: 24/09/2020 - Fecha de aceptación: 6/02/2021 - Fecha de publicación en línea: 05/04/2021 Correspondencia: Rami Mikler, Carrera 7ª No 40-62, Hospital Universitario San Ignacio, Bogotá. D.C. Colombia. Teléfono: 3102080869. Correo electrónico: rmikler@husi.com.co

Citar como: Mikler R, Pinzón FE, Metke R. Cirugía bariátrica secundaria en el Hospital Universitario San Ignacio Rev Colomb Cir. 2021;36:457-61. https://doi.org/10.30944/20117582.752

Este es un artículo de acceso abierto bajo una Licencia Creative Commons - BY-NC-ND https://creativecommons.org/licenses/by-ncnd/4.0/deed.es 


\begin{abstract}
Introduction. The World Health Organization defines obesity as an increase in the percentage of body fat, generally accompanied by an increase in weight, the quantity and distribution of which determine the health of the individual. It is characterized by being a chronic disease and very difficult to manage. Bariatric surgery is an increasingly frequent procedure, which has proven to be the best therapeutic option for the management of moderate and severe obesity, a disease that has been gaining ground in the world. According to the 2015 National Survey of the Nutritional Situation in Colombia, the incidence of overweight was $37.8 \%$ and obesity was $18.7 \%$. With the increase in primary bariatric surgery, the frequency of revision, conversion and reversal bariatric surgery has also increased, with conversion being the most frequent.
\end{abstract}

Methods. Retrospective observational study in which patients underwent bariatric conversion surgery at the San Ignacio University Hospital between 2014 and 2019 were analyzed.

Results. In our institution, conversion surgery corresponds to $8.8 \%$, and coincides with the world statistics for this procedure. The most frequent cause, unlike the literature, is gastroesophageal reflux, followed by weight gain.

Discussion. The results obtained at the San Ignacio University Hospital are similar to the world figures for conversion procedures. Resolution of gastroesophageal reflux symptoms and proper management of weight gain are obtained in a high percentage of patients, with very few complications.

Keywords: obesity; bariatric surgery; secondary; conversion; gastroesophageal reflux; weight loss.

\section{Introducción}

La obesidad es una epidemia cuyas cifras se vienen incrementando de forma acelerada. En la población de Norte América, la prevalencia para 2014 fue del 36,4 \%, doblando la prevalencia desde $1980^{1}$. En Colombia, según la Encuesta Nacional de Situación Nutricional (ENSIN), para 2015 la prevalencia de sobrepeso fue del 37,8 \%, y del 18,7 \% para la obesidad, es decir un total de exceso de peso del $56,5 \%{ }^{2}$.

La cirugía bariátrica ha evolucionado y en la actualidad se considera el método más efectivo para el tratamiento de ciertos tipos de obesidad, ya que logra la perdida de la mayor cantidad de peso en los pacientes, permite mantener un bajo peso y corrige la mayoría de las comorbilidades ${ }^{3}$. Actualmente, más del $80 \%$ de la cirugía realizada en todo el mundo corresponde a la manga gástrica y al baipás gástrico en $Y$ de Roux. El mini baipás gástrico (MGB, por sus siglas en inglés) es la tercera técnica en frecuencia y la de mayor crecimiento en los últimos años ${ }^{4-7}$. A pesar de la efectividad de estas cirugías, se pueden presentan complicaciones y reganancia de peso ${ }^{8}$.

Con el aumento en el número de cirugías bariátricas realizadas, también se ha incrementado el número de cirugías secundarias ${ }^{9}$. En Estados Unidos de América se menciona una tasa de revisión o conversión de procedimientos como banda gástrica en un $26 \%$, manga gástrica en un 9,8\% y baipás gástrico en un $4,9 \%{ }^{10}$. La prevalencia del procedimiento de conversión ha aumentado significativamente en los últimos años, con un porcentaje en 2016 del 13,9\% de un total de cerca de 216.000 cirugía realizadas, duplicando la cantidad de las intervenciones realizada en 2012, cuando se registró solo un $6 \%{ }^{1,9}$.

El objetivo de este estudio fue presentar una serie de casos de procedimientos bariátricos realizados en el Hospital Universitario San Ignacio, en los últimos años y la tasa de cirugías de conversión. Además, evaluar las causas de la cirugía de conversión y los desenlaces posteriores a la misma.

\section{Métodos}

Estudio observacional retrospectivo. Se revisó la historia clínica de todos los pacientes del Hospital Universitario San Ignacio que fueron sometidos a algún procedimiento bariátrico entre 2014 y 2019. La muestra final estuvo conformada por los pacientes que requirieron cirugía de conversión. 
Los criterios de inclusión fueron: pacientes mayores de 18 años con obesidad. Se realizó un estudio de las variables asociadas al procedimiento quirúrgico, cirugía realizada inicialmente, tipo de cirugía (revisión o conversión), complicaciones, y seguimiento de peso y de índice de masa corporal (IMC).

\section{Resultados}

Entre 2014 y 2019 , se realizaron un total de 484 procedimientos bariátricos, y en el 8,8 \% de ellos $(n=43)$ fue necesario realizar cirugía de conversión. El rango de edad varió entre 31 y 67 años, con un promedio de edad de 54 años. El tiempo transcurrido entre la cirugía inicial y el procedimiento de conversión estuvo entre los 2 y los 12 años, con un promedio de 4,4 años.

En el 46,5\% de los casos ( $n=20$ ) la conversión se realizó debido a la presencia de reflujo gastroesofágico (RGE), en el 13,9\% $(n=6)$ por reganancia de peso, en $32,5 \%$ de los pacientes $(n=14)$ por reganancia de peso y presencia de RGE, en un 2,3\% (n=1) por un mal control metabólico y reflujo gastroesofágico y un 4,6\% $(n=2)$ por otras causas (un caso de obstrucción gástrica y uno de desnutrición severa). En general, las dos principales causas de conversión fueron RGE en un $79 \%$ $(n=34)$, y reganancia de peso en un $46,5 \%(n=20)$ de los pacientes. En un paciente con desnutrición severa fue necesario realizar cirugía de baipás gástrico a anatomía usual.
De los pacientes que presentaban solamente RGE, el $35 \%$ tenían como procedimiento previo una manga gástrica, un $25 \%$ una gastro-gastroplastia y un $40 \%$ un mini baipás. De los pacientes con reganancia de peso, a 17 \% se les había practicado banda gástrica, $17 \%$ gastro-gastroplastia, un $33 \%$ mini baipás y al $33 \%$ una manga gástrica. De los pacientes con reganancia de peso y RGE un $7 \%$ tenían una gastro-gastroplastia, un $50 \%$ manga gástrica y un $43 \%$ mini baipás. Del total de pacientes con RGE, un $32 \%$ tenían mini baipás y $32 \%$ manga gástrica. Del total de pacientes con reganancia de peso un $40 \%$ tenían mini baipás y un $45 \%$ manga gástrica.

El seguimiento posterior al procedimiento quirúrgico fue mayor de dos años en el 53,5 \% $(n=23)$ de los pacientes, y menor en el 46,5\% $(n=20)$ de los casos (cuatro pacientes seguimiento de 2 meses, 10 pacientes con seguimiento de 6-9 meses y seis pacientes con seguimiento de 12-18 meses).

La mejoría del REG y la corrección de la reganancia de peso se logró en la mayoría de los pacientes (figura 1). El $20 \%$ de los pacientes con reganancia de peso perdieron menos del $10 \%$ de su exceso de peso, y la pérdida del exceso de peso a través del tiempo fue de $37,8 \%$ en el primer mes ( 20 de 20 pacientes), $52 \%$ al sexto mes (17 de 20 pacientes), $35,5 \%$ a los 12 meses (12 de 20 pacientes) y $34,2 \%$ a los 24 meses ( 5 de 20 pacientes).

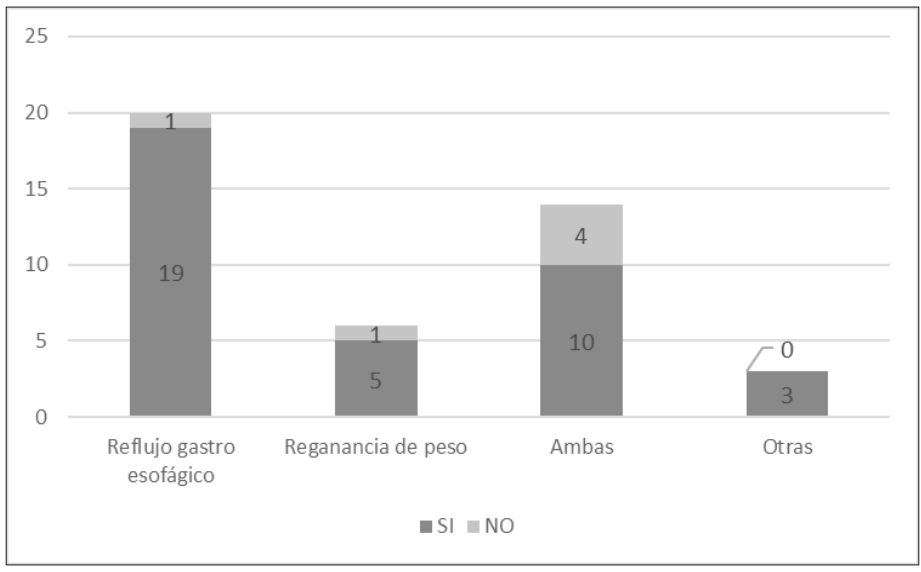

Otras: mal control metabólico, obstrucción gástrica y desnutrición severa

Figura 1. Resolución de los síntomas en los pacientes a quienes se les realizó cirugía bariátrica de conversión. 
El seguimiento del reflujo en los pacientes sintomáticos se realizó mediante estudios imagenológicos (tomografía), fisiológicos ( $\mathrm{pH}$-metria de 24 horas) y endoscópicos, que confirmaron solo 2 casos de persistencia de RGE.

Se presentaron solo tres complicaciones tempranas $(6,9 \%)$, un paciente con sangrado que requirió transfusión, un caso de perforación contenida y una fistula del bastón yeyunal, que fueron reintervenidos. Las complicaciones tardías ocurrieron en cuatro pacientes $(9,3 \%)$, una hernia de Petersen, una hernia hiatal persistente, una eventración por un puerto epigástrico y una estenosis tratada con dilatación con balón. La estancia hospitalaria fue en promedio de 4,2 días.

\section{Discusión}

La cirugía bariátrica secundaria (revisión, conversión y reversión) es un procedimiento que viene en aumento de forma paralela al incremento de la cirugía bariátrica primaria ${ }^{1}$. De acuerdo con la literatura mundial, varía entre el 5-60 \% dependiendo de la técnica primaria, con un promedio de $13,4 \%{ }^{9}$, cercano al encontrado en nuestro estudio, donde la cirugía secundaria correspondió al 8,8 \% del total de la cirugía bariátrica.

En nuestra institución las causas más frecuentes de cirugía secundaria fueron el RGE y la reganancia de peso, siendo la primera la principal causa, a diferencia de lo informado en literatura mundial, en donde la reganancia de peso ocupa el primer lugar.

Los procedimientos de reoperación se consideran una cirugía de alta complejidad, sin embargo, en nuestra institución se encontró un bajo porcentaje de complicaciones, tanto tempranas como tardías y, en ningún caso se presentó mortalidad.

Llama la atención el alto índice de RGE encontrado en nuestros pacientes con mini baipás gástrico, que no se compagina con lo descrito en la literatura mundial. Probablemente pudo haberse debido a poca rigurosidad en los detalles de la técnica quirúrgica, principalmente en cuanto a la longitud del reservorio gástrico.

Es importante mencionar que para definir llevar a un paciente a una cirugía de conversión por
RGE, este debe ser estudiado minuciosamente con todos los exámenes de fisiología y anatomía esófago-gástrica, los cuales deben repetirse en el seguimiento posoperatorio. Tanto los pacientes como la técnica de conversión se deben seleccionar de forma correcta para lograr resultados adecuados.

\section{Conclusiones}

Consideramos que los resultados obtenidos en el Hospital Universitario San Ignacio, Bogotá, D.C., Colombia, se asemejan a los estándares mundiales de procedimientos de conversión. A nivel mundial, la reganancia de peso es considerada la principal indicación de la cirugía secundaria bariátrica, pero en nuestro estudio encontramos que el RGE refractario al tratamiento médico es la causa más frecuente de conversión, seguido de la reganancia de peso. La resolución de los síntomas de RGE y el manejo adecuado de la reganancia de peso, se obtiene en un muy alto porcentaje de pacientes, con un bajo porcentaje de complicaciones, a pesar de ser una técnica de alta complejidad.

\section{Cumplimiento de normas éticas}

Consentimiento informado: este estudio es una revisión retrospectiva de historias clínicas, y como tal, no hay necesidad de un consentimiento informado. El Comité de Ética Institucional aprobó el diseño y la metodología del estudio.

Declaración de conflictos de interés: los autores declaran que no tienen conflictos de interés.

Fuentes de financiación: Recursos propios de los autores.

\section{Contribución de los autores}

Concepción y diseño del estudio: Rami Mikler, Fabio Eduardo Pinzón C, Ricardo Metke

Adquisición de datos: Rami Mikler, Fabio Eduardo Pinzón C, Ricardo Metke

Análisis e interpretación de datos: Rami Mikler, Fabio Eduardo Pinzón C, Ricardo Metke

Redacción del manuscrito: Rami Mikler, Fabio Eduardo Pinzón C, Ricardo Metke

Revisión crítica: Rami Mikler, Fabio Eduardo Pinzón C, Ricardo Metke 


\section{Referencias}

1. Phillips BT, Shikora SA. The history of metabolic and bariatric surgery: Development of standards for patient safety and efficacy. Metabolism. 2018;79:97-107. https://doi.org/10.1016/j.metabol.2017.12.010

2. Guevara CE, Mikler R, editores. Guías de manejo en cirugía bariátrica y metabólica 2018. 2da. Edición Actualización 2018. Bogotá: Distribuna; 2018.

3. Le Roux CW, Heneghan HM. Bariatric Surgery for Obesity. Med Clin North Am. 2018;102(1):165-82. https://doi.org/10.1016/j.mcna.2017.08.011

4. Shoar S, Saber AA. Long-term and midterm outcomes of laparoscopic sleeve gastrectomy versus Roux-en-Y gastric bypass: a systematic review and meta-analysis of comparative studies. Surg Obes Relat Dis. 2017;13:170-80.

https://doi.org/10.1016/j.soard.2016.08.011.

5. Li J, Lai D, Wu D. Laparoscopic Roux-en-Y gastric bypass versus laparoscopic sleeve gastrectomy to treat morbid obesity-related comorbidities: a systematic review and meta-analysis. Obes Surg. 2016;26(2):429-42. https://doi.org/10.1007/s11695-015-1996-9

6. Zhang Y, Zhao H, Cao Z, Sun X, Zhang Ch, Cai W, et al. A randomized clinical trial of laparoscopic Roux-en-Y gastric bypass and sleeve gastrectomy for the treatment of morbid obesity in china: a 5-year outcome. Obes Surg. 2014;24:1617-24.

https://doi.org/10.1007/s11695-014-1258-2

7. Versteegden DPA, Van himbeeck MJ, Nienhuijs SW. Improvement in quality of life after bariatric surgery: sleeve versus bypass. Surg Obes Relat Dis. 2018;14:170-74. http://dx.doi.org/10.1016/j.soard.2017.10.008

8. Creange C, Jenkins M, Pergamo M, Fielding G, Ren-Fielding C, Schwack B. Gastric band conversion to Rouxen-Y gastric bypass shows greater weight loss than conversion to sleeve gastrectomy: 5-year outcomes. Surg Obes Relat Dis. 2018;14:1531-6. https://doi.org/10.1016/j.soard.2018.06.002

9. English WJ, DeMaria EJ, Brethauer SA, Mattar SG, Rosenthal RJ, Morton JM, American Society for Metabolic and Bariatric Surgery estimation of metabolic and bariatric procedures performed in the United States in 2016. Surgr Obes Relat Dis. 2018;14:259-263. https://doi.org/10.1016/j.soard.2017.12.013

10. Altieri MS, Yang J, Nie L, Blackstone R, Spaniolas K, Pryor A. Rate of revisions or conversion after bariatric surgery over 10 years in the state of New York. Surg Obes Relat Dis. 2018;14:500-8.

https://doi.org/10.1016/j.soard.2017.12.019 